\title{
Community defibrillation in the Scottish Highlands
}

\author{
M.C. Jones, F. Kerr and A.P.R. Eckersley ${ }^{1}$ \\ Department of Medicine, Raigmore Hospital, Inverness IV2 $3 U$ J and ${ }^{1} 12$ Crown Avenue, Inverness IV2 \\ $3 N F, U K$.
}

\begin{abstract}
Summary: Prompt defibrillation after cardiac arrest is necessary to save life. Inexpensive systems providing defibrillation facilities in the community of the Highlands have been devised, one for Inverness town practices, and one for rural practices. Both systems work by 'blind' defibrillation, once the clinical diagnosis of cardiac arrest is confirmed. Acute anti-arrhythmic drugs and intravenous infusions were not available. The systems functioned satisfactorily with general acceptance by the general practices concerned.
\end{abstract}

\section{Introduction}

One hundred and fifty thousand patients die annually from coronary artery disease in the United Kingdom, 55,000 being under the age of 70 years. Over $60 \%$ of these patients die within the first hour of the onset of their symptoms and thus are likely to die outside hospital. Ninety per cent of the deaths occur as a result of ventricular fibrillation. ${ }^{1}$ In an attempt to overcome these high immediate mortality figures Pantridge and colleagues introduced the first pre-hospital coronary care facility in Belfast in $1966 .^{2}$ This development soon demonstrated that resuscitation from ventricular fibrillation could be achieved before the patient reached hospital ${ }^{3}$ and indeed, in patients aged 70 or less treated for acute autonomic disturbances within the first hour hospital mortality was only $9 \%$ and cardiogenic shock was rare. ${ }^{4}$

The adoption of mobile coronary care units has since become widespread in many countries and such units are highly developed in the United States. Staffing in these units, especially in the United States, has been mainly by paramedical workers and/or technicians ${ }^{5}$ and in some centres, most notably Seattle, the efficacy of these services has been enhanced by training approximately $\frac{1}{3}$ of the adult population in cardio-pulmonary resuscitation. ${ }^{6}$ In the United Kingdom, however, prehospital coronary care has not become widely developed despite the recommendations of the Joint Working Party of the Royal College of Physicians of London and the British Cardiac Society published in $1975 .^{7}$ The failure of the Department of Health to implement the recom-

Correspondence: M.C. Jones, M.R.C.P., Department of Medicine and Therapeutics, Polwarth Building, Foresterhill, Aberdeen AB9 2ZD, UK.

Accepted: 8 September 1989 mendations was partly based on the studies reported from Nottingham in 1976 which showed that a specially equipped mobile coronary care unit was unlikely to be sufficiently effective in reducing mortality ${ }^{8}$ although experience in Brighton suggested that defibrillator-equipped ambulances staffed by ambulance-men trained to treat common arrhythmias could be successful. ${ }^{9}$ Development has also been inhibited by the high capital and revenue costs.

Recognizing that prompt defibrillation is necessary to save life we designed an inexpensive system of community defibrillation. 'Blind' defibrillation undertaken without electrocardiographic monitoring of patients suffering from cardiac arrest has been proposed and found to be effective in the community. ${ }^{10}$ In the United Kingdom we have a uniquely comprehensive system of community medical care provided by general practitioners; particularly in remote rural areas patients usually call their general practitioner (GP) first. This is an important early link in the chain which should not be ignored. Two recent reports have described studies where single practices were equipped with a portable defibrillator which was carried by the on-call partner for the day. ${ }^{10,11}$ We describe two alternate schemes for community defibrillation by general practitioners.

\section{Methods}

Two systems were evaluated. A town system based around Inverness involved 8 practices with a total population of 45,900. Two of the town ambulances were equipped with a portable defibrillator (Light Guard Mini Defibrillator - Temtech Ltd, N. Ireland). Each practice nominated one partner each day to be responsible for answering chest pain 
and collapse calls. This partner carried a British Telecom radiopager and whenever it was suspected that a patient was suffering from a possible myocardial infarction contact was made simultaneously with one of the ambulances carrying a defibrillator. Hence, on arrival at the patient's home, the GP had access to a defibrillator and, furthermore, had the skilled assistance of two ambulance-men for cardiopulmonary resuscitation. If it was found that the patient required transport to hospital by ambulance he was accompanied by the GP in case defibrillation was needed. The GP was subsequently transported back to his car by the ambulance crew.

The country study involved two practices centred on Grantown-on-Spey and Ullapool (total population 6,580 ). In both practices the partner on-call carried the portable defibrillator with him and only called the ambulance when transfer of the patient to hospital was required. Because of the distance involved it was not possible for these GPs to routinely accompany their patients to hospital. Consequently defibrillator cover for most patients occurred only while the GP was in the patient's home or in the immediate transfer from home to ambulance. All Inverness call-outs, where the general practitioner requested the defibrillator, were recorded over the period June 1st 1985-May 31st 1987 . The country practices provided details during the same period only when the defibrillator was used. At the end of this study period a questionnaire was sent to each Inverness practice to assess any difficulties experienced during the study and to ascertain whether the practitioners felt the scheme should continue and/or should be modified. The study was approved by the Highland Health Board Ethical Committee.

\section{Results}

During the study period the ambulance defibrillator was called upon 49 times by the practices within Inverness. The defibrillator was used during 4 of these call-outs. On each occasion the defibrillator was used in the patient's home and on no occasion was defibrillation required whilst the patient was being transported by ambulance to hospital. Defibrillation was successful on one occasion but the patient died on his sixth hospital day. Forty three patients were transported to hospital with the GP in attendance, 6 patients were left at home, 3 who were judged not to have suffered a myocardial infarction and 3 who were declared dead after failed defibrillation. Of the 43 patients transported to hospital the working diagnosis in each case was acute myocardial infarction. All patients were taken to Raigmore Hospital, a district general hospital with acute coronary care facilities. During the study period a total of 151 cases of myocardial infarction (taken from Scottish Medical Records 1 [SMR1] returns) were admitted from the same practices in Inverness.

The results from the rural practices showed that 5 patients were defibrillated and transported to Hospital, 2 were successfully defibrillated in the patient's home, 2 immediately on entering the ambulance and one in the local cottage hospital. Thereafter, 2 patients died on their fourth hospital day suffering from cardiogenic shock. One died during a subsequent admission three weeks later and 2 are still alive with no demonstrable neurological or intellectual sequelae. During the study period 54 cases of myocardial infarction (SMR1 returns) were referred for admission by doctors working in these practices.

The collapse-defibrillation interval for the 4 patients treated by doctors working within Inverness was recorded in only 2 of the cases. In one of these, immediate defibrillation was attempted and in the other the interval was approximately 8 minutes. In the 5 episodes of attempted defibrillation performed by the doctors working in Grantown and Ullapool the median collapsedefibrillation interval was two minutes (range: immediate defibrillation to 5 minutes). This was based on estimates supplied by the GP attending at the time of cardiac arrest.

All of the Inverness practices responded to the end of study questionnaire. All practices felt that a community defibrillation scheme should continue but five of the seven thought that the scheme would be improved if ambulancemen were trained to defibrillate on their own initiative. Two of the practices were undecided rather than opposed to this suggestion. Only one practice was concerned about the lack of electrocardiographic monitoring and two were undecided. One practice would have preferred the availability of intravenous infusion and antiarrhythmic drugs.

\section{Discussion}

Expensively equipped mobile coronary care ambulances may be justified in areas of high population density but they are impractical in smaller towns and rural areas. A study of the management of myocardial infarction by general practitioners not equipped with defibrillators between 1978 and 1980 found that 111 of 511 patients suspected of suffering from acute myocardial infarction died within 48 hours of the onset of chest pain. Twentythree of these died in the presence of the general practitioner. ${ }^{12}$ At that stage it was suggested that general practitioners should be supplied with defibrillators. A subsequent study from a community with defibrillator equipped GPs showed 
that of 54 patients who collapsed with a cardiac arrest either in the presence of their general practitioner or less than 5 minutes before the doctor's arrival, 32 had cardiac output re-established and 22 were subsequently discharged alive from hospital. ${ }^{10}$ A more recent study showed that $28 \%$ of patients who had a cardiac arrest in the presence of the general practitioner and were defibrillated survived to leave hospital. ${ }^{11}$ Equipping GP practices with a portable defibrillator has thus been found to be successful in some areas.

We evaluated a scheme whereby two ambulances in a moderate sized town (population 45,900) were equipped with a portable defibrillator and were required to attend quickly the patient's home when requested by the GP. We recognized that in the smaller remote towns and villages of the Highlands usually served by one group of GPs the sensible decision was to equip the individual practices. The two peripheral practices studied were situated 34 miles (Grantown) and 84 miles (Ullapool) from Inverness.

The SMR1 returns of acute myocardial infarction rely on discharge diagnosis and therefore offer only an approximation of the numbers of possible myocardial infarctions seen by the general practitioners and referred to hospital. All patients from rural practices who were suspected of suffering from an acute myocardial infarction were seen by a general practitioner equipped with a defibrillator. In these practices GPs are able to spend the first critical hours with their patients and only under special circumstances is admission to hospital required. In this particular study 5 post defibrillation patients were transferred to Raigmore Hospital with their GPs in attendance.

The returned forms suggested that approximately one-third of the town patients with myocardial infarction were covered by the availability of a defibrillator.

Thus the defibrillator was used in 4 of 49 call-outs in Inverness and on 5 occasions in the rural practices during a period when SMR 1 returns indicated 54 acute myocardial infarctions were admitted. The rural system where each practice was equipped with a defibrillator, therefore, seemed more successful. The reason for this appears to have been a reluctance by some of the town GPs to call for the defibrillator ambulance. This was reflected in replies to the questionnaire where 2 practices indicated that they would have preferred their own defibrillator while 2 practices were undecided on this point. Despite the obvious deficiencies both systems met with relative success. Six patients in total were successfully defibrillated, two of whom are still surviving.

Five of the seven practices felt that the scheme would be improved if ambulancemen were trained to defibrillate on their own initiative and, possibly, this reflects a disinclination on the part of the general practitioners to develop a practice based defibrillation service. The presence of ambulancemen trained in defibrillation would certainly have the advantage of including patients using the emergency 999 telephone system. The use of semiautomatic defibrillators by ambulancemen has now been approved by the Department of Health and the Scottish Home and Health Department. This is likely to be a much more costly scheme because of the more sophisticated equipment and the need to train ambulancemen in the use of such specialized equipment.

The decision to avoid intravenous infusion and antiarrhymthmic drugs seems vindicated by the fact that all of the patients successfully defibrillated reached hospital without further need for defibrillation and experienced no important rhythm disturbance at the time of admission to hospital.

Data from Nottingham where the training programme for ambulance crew does not include endotracheal intubation, intravenous infusion or drug administration but does include defibrillator usage demonstrate that 'blind' defibrillation can make a difference in the overall mortality from myocardial infarction where 44 of 403 consecutive patients suffering cardiac arrest were managed by ambulance crews and survived to leave hospital. ${ }^{13}$

This study demonstrates that general practitioners, using simple inexpensive defibrillators without electrocardiographic monitoring or antiarrhythmic drugs, can successfully resuscitate patients from cardiac arrest following myocardial infarction.

\section{References}

1. Adgey, A.A.J. Coronary patient - early treatment? Br Heart J 1980, 44: 357-360.

2. Pantridge, J.F. \& Geddes, J.S. Cardiac arrest after myocardial infarction. Lancet 1966, i: 807-808.

3. Adgey, A.A.J., Nelson, P.G., Scott, M.E. et al. Management of ventricular fibrillation outside hospital. Lancet 1969, i: 1169-1171.

4. Geddes, J.S. Twenty years of prehospital coronary care. $\mathrm{Br}$ Heart J 1986, 56: 491-495.

5. Redmond, A.D. Paramedics in the United Kingdom? Br Med $J$ 1984, 288: 622-623.

6. Thompson, R.G., Hallstrom, A.P. \& Cobb, L.A. Bystander initiated cardiopulmonary resuscitation in the management of ventricular fibrillation. Ann Int Med 1979, 90: 737-740.

7. Royal College of Physicians of London and The British Cardiac Society. The care of the patient with coronary heart disease. Report of a joint working party. $J \boldsymbol{R}$ Coll Physicians. Lond 1975, 10: 5-46.

8. Hampton, J.R., Dowling, M. \& Nicholas, C. Comparison of results from a cardiac ambulance manned by medical or non-medical personnel. Lancet 1977, i: 526-529. 
9. Mackintosh, A.F., Crabb, M.E., Grainger, R. Williams, J.H. \& Chamberlain, D.A. The Brighton resuscitation ambulances: review of 40 consecutive survivors of out-ofhospital cardiac arrest. $\mathrm{Br}$ Med J 1978, 1: 1115-1118.

10. Clyde, C., Kerr, A., Varghese, A. \& Wilson, C. Defibrillators in general practice. $\mathrm{Br}$ Med J 1984, 289: 1351-1353.

11. Pai, G.R., Haites, N.E., Rawles, J.M. One thousand heart attacks in Grampian: the place of cardiopulmonary resuscitation in general practice. Br Med J 1987, 294: 352-354.
12. Rawlins, D.C. Study of the management of suspected cardiac infarction by British immediate care doctors. Br Med J 1981, 282: $1677-1679$.

13. Rowley, J.M., Mounser, P., Garner, C. \& Hampton, J.R. Advanced training for ambulance crews: implication from 403 consecutive patients with cardiac arrest managed by crews with simple training. Br Med J 1987, 295: 1387-1389. 